\title{
Assessment of cardiac function by pulse wave analysis. A computational study
}

Francesco Piccioli ( $\nabla$ francesco.piccioli@unife.it)

University of Ferrara https://orcid.org/0000-0003-1594-7478

Alessandro Valiani

University of Ferrara Faculty of Engineering: Universita degli Studi di Ferrara Dipartimento di Ingegneria Jordi Alastruey

King's College London School of Biomedical Engineering and Imaging Sciences

Valerio Caleffi

University of Ferrara Department of Engineering: Universita degli Studi di Ferrara Dipartimento di Ingegneria

\section{Research Article}

Keywords: Cardiovascular network, Ventricular-arterial coupling, Haemodynamic indices, Pulse wave morphology, Sensitivity analysis, Left ventricular contractility, Left atrial contractility, Heart valves dynamics, PPG signal

Posted Date: February 24th, 2022

DOI: https://doi.org/10.21203/rs.3.rs-1367216/v1

License: (c) (1) This work is licensed under a Creative Commons Attribution 4.0 International License. Read Full License 


\title{
Assessment of cardiac function by pulse wave analysis.
}

\section{A computational study}

\author{
Francesco Piccioli ${ }^{1}$ Alessandro Valiani ${ }^{1}$ Jordi Alastruey $^{2,3}$ Valerio Caleffi $^{1}$
}

February 16, 2022

10

correspondence: Francesco Piccioli, Department of Engineering, University of Ferrara, Via G. Saragat 1, 44122 Ferrara, Italy e-mail: francesco.piccioli@unife.it 


\begin{abstract}
The development of methodologies that provide a comprehensive assessment of cardiac function from pulse wave analysis in the systemic circulation could avoid current practice using expensive, invasive and operator-dependent measurement techniques. This work addressed this topic computationally. A cardiovascular model, constituted of a lumped-parameter model of the left part of the heart coupled to a one-dimensional model of the arterial network, was validated using reference pulse waveforms in turn verified by comparison with in vivo measurements. The validation was specifically designed to verify the performance of the model in returning physiological pulse waves for assigned cardiac properties. Successively, a sensitivity analysis was performed to assess the effects of variations in cardiac parameters on central and peripheral pulse waves. Results show that left ventricle contractility, stroke volume, cardiac cycle duration and heart valves impairment are determinants of central waveforms morphology, pulse pressure and its amplification. Contractility of the left atrium has negligible effects on vascular pulse waves. Results also suggest that it may be possible to infer left ventricular dysfunction by analysing the timing of the dicrotic notch. This study suggests which cardiac properties can be extracted from central and peripheral pulse waves to assess cardiac function.
\end{abstract}

keywords: Cardiovascular network; Ventricular-arterial coupling; Haemodynamic indices; Pulse wave morphology; Sensitivity analysis; Left ventricular contractility; Left atrial contractility; Heart valves dynamics; PPG signal 


\section{Introduction}

Pathologies affecting cardiac function are responsible for morbidity and mortality worldwide ${ }^{12}$. Cardiac haemodynamics properties are of paramount importance for the assessment of cardiac function and, hence, cardiovascular risk. Cardiac function is usually assessed invasively. For example, left ventricular filling pressure - which is used to assess left ventricular function - can be measured directly by placing a catheter in the proximal aorta or the left ventricle, or indirectly from the pulmonary artery $39 \mid 34$. This is an expensive and time-consuming procedure that carries risk to patients (e.g., blood clot formation and embolization) due to its invasive nature, even when performed in specialized centres ${ }^{22}$.

An indirect estimation of cardiac properties by pulse wave analysis may overcome these obstacles. Currently, pulse wave analysis is usually employed to obtain information on vascular properties, such as arterial stiffness ${ }^{26}$, but it has the potential to provide information on cardiac function, since changes in cardiac properties affect the morphology of pulse wave signals measured in the vasculature ${ }^{14}$. Nowadays, pulse waves can be acquired noninvasively by wearable devices which are more convenient and less expensive for large-scale screening than invasive exams. In particular, the photoplethysmogram (PPG) pulse wave is easily acquired using pulse oximeters, which are frequently used in healthcare settings to measure arterial blood oxygen saturation and pulse rate. PPG signals can also be acquired by devices available to the wider population, such as smartphones, smartwatches and fitness bands $\frac{45 \mid 10}{\text {. }}$

Databases of in silico pulse waves signals representative of cohorts of real subjects can be produced using robust and efficient computational blood flow models for the development and pre-clinical testing of pulse wave analysis algorithms 1146 . Virtual subjects are characterised by haemodynamic variables spanning the physiological range, even in disease-related conditions ${ }^{15135}$. Different numerical models can be employed to accomplish this task: zerodimensional (0-D) lumped-parameters models for simulating blood flow in distal vessels ${ }^{8}$ and specific organs such as the heart $\frac{25}{25}$, one-dimensional (1-D) models for simulating blood flow in the large arteries of the human circulation ${ }^{33}$, and three-dimensional (3-D) models ${ }^{[15}$, 
which are often used to simulate blood flow in localised regions of the vasculature, such as arterial bifurcations, due to their high computational cost. 1-D models can simulate pulse wave signals with a reasonable accuracy compared with both 3 -D models $s^{\sqrt{47115}}$ and experimental data ${ }^{21}$, and at much lower computational cost compared to 3-D models. They have, therefore, been used to generate databases of pulse waves for thousands of virtual subjects.

The aim of this study is to investigate the effect of cardiac properties on pulse wave morphology using a 1-D model of the arterial vasculature ${ }^{\sqrt{33}}$ coupled to a 0 -D model of cardiac contraction. The 1-D model considers the viscoelastic behaviour of vessel walls ${ }^{315}$. The heart model is based on the state-of-the-art model by Mynard et al. 25128 . The ability of our model to describe physiological pulse waves for assigned cardiac properties is verified using reference pulse waveforms ${ }^{2818}$. A sensitivity analysis (SA) is then performed to study the effect of all cardiac model parameters on central and peripheral pulse waveforms and the PPG signal in the digital artery.

\section{Materials and Methods}

\subsection{Cardiovascular model}

A schematic representation of the proposed cardiovascular model is shown in Fig. 1. It includes three key components. First, the 1-D model arterial network consists of 116 arterial segments representing the largest arteries of the thorax, limbs and head ${ }^{8}$. Arterial segments were modelled as thin viscoelastic tubes of linearly tapered diameter. Second, the RCR Windkessel model was employed at the terminal vessels as outlet boundary condition (BC), to describe the resistance and compliance of peripheral vascular beds. Third, a cardiac contraction model representing the left-side heart was coupled to the vascular network as inlet BC. 

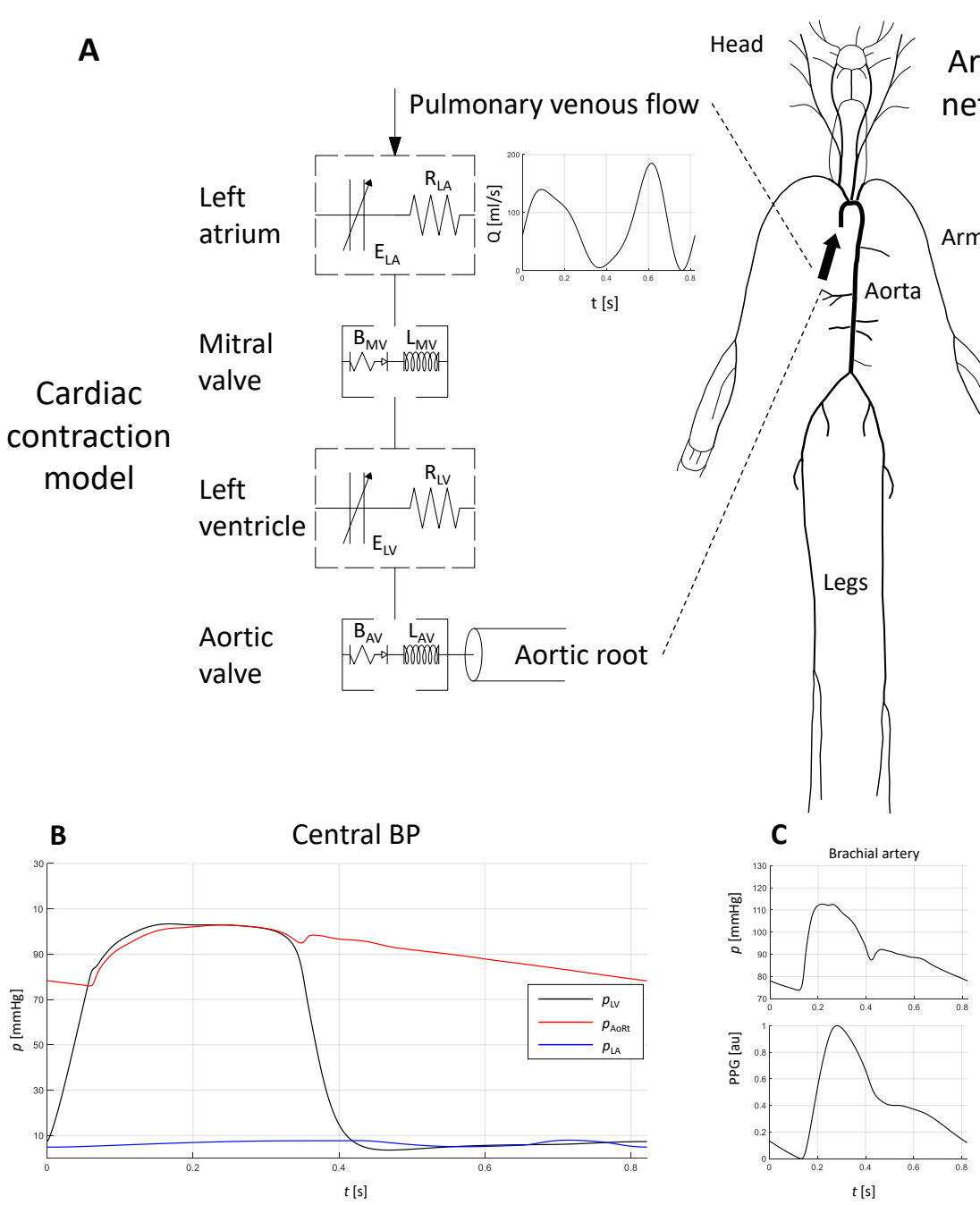

Arterial
network

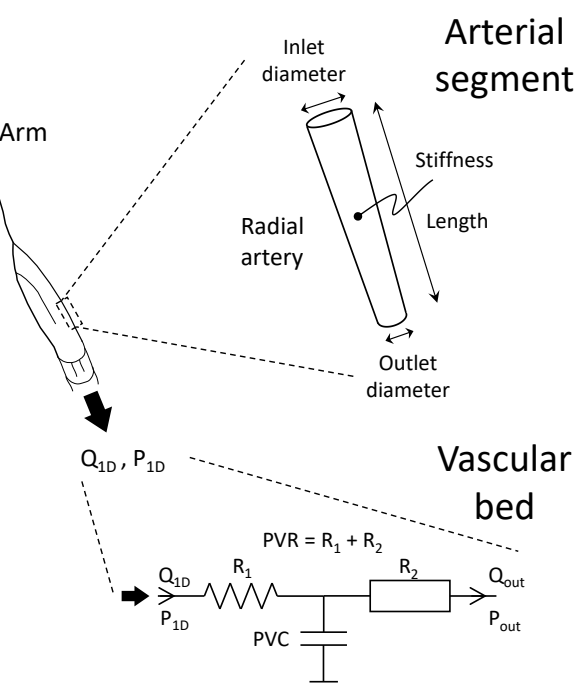

Figure 1: The schematic representation of the 1-D model vascular network coupled with the 0-D cardiac contraction model (A), central blood pressure waveforms (B), peripheral blood pressure waveforms (C). The cardiovascular network consists of the arterial tree, containing the 116 largest systemic arteries, the left-side heart coupled to the vascular network at the aortic root, and lumped parameter models at 1-D model terminal segments representing the vascular bed. See text for the definition of parameters.

\subsubsection{Vascular model}

The 1-D model vascular network was modelled using the augmented fluid-structure interaction (a-FSI) system ${ }^{3512}$, a hyperbolic set of equations. This includes equations for the conservation of mass and momentum, and a closure equation relating vessel cross-sectional 
area and internal pressure, the so-called tube law $\underline{43}$. Key assumptions for the haemodynamic model are: laminar flow, incompressible and Newtonian fluid (blood density, $\rho=1060 \mathrm{~kg} / \mathrm{m}^{3}$; blood viscosity, $\mu=2.5 \mathrm{mPa} \mathrm{s}$ ), and no energy losses at bifurcations.

For the geometrical and mechanical characteristics of the network, the reader is referred to Charlton et al. ${ }^{8}$. The viscoelastic formulation of the tube law was described using the Standard Linear Solid Model (SLSM) 16113 , entailing the presence of a stiff term in the governing system of equations. Therefore, the numerical scheme was chosen to work efficiently with all ranges of relaxation time. It consists of the stiffly accurate Implicit-Explicit (IMEX) strong-stability-preserving $\operatorname{SSP}(3,3,2)$ scheme, characterised by three stages for both the implicit and explicit parts and second-order accuracy ${ }^{32 / 3}$. A Finite Volume (FV) method was used for the discretization in space. The treatment of internal BCs, i.e. vessel junctions, and external BCs followed the methodology presented by Piccioli et al. ${ }^{33}$.

\subsubsection{Left-side cardiac model}

The cardiac contraction model includes the left atrium (LA), the left ventricle (LV), and the adjacent valves, i.e. mitral valve (MV) and aortic valve (AV). The pulmonary venous flow rate (PVFR) entering the LA was prescribed as a function in time. The cardiac contraction model was employed as inlet BC for the vascular model, as described in Sect 2.1.3.

The LA and LV were modelled through the time-varying elastance function $E(t)^{\sqrt{25}}$. Mimicking the myocardium contraction, elastance changes between its extrema, relating the pressure in the cavity $p(t)$ with the cavity volume $v(t)$ by

$$
p(t)=E(t)\left[v(t)-v_{p 0}\right]\left[1-K_{s} q_{\text {out }}(t)\right]
$$

where $v_{p 0}$ is the unstressed volume, $K_{s}$ is a resistive term, and $q_{\text {out }}(t)$ is the chamber outflow 25 . The elastance $E(t)$ was defined by the 'double-Hill' function $25 \mid 20$

$$
E(t)=\left[\frac{E_{\text {max }}-E_{\text {min }}}{\max \left(H_{1}(t) H_{2}(t)\right)}\right] H_{1}(t) H_{2}(t)+E_{\text {min }},
$$


where $E_{\min }$ and $E_{\max }$ are the minimal and maximal values of the elastance, respectively. $E_{\min }$ governs diastolic passive stiffness, hence the filling phase of the chamber ${ }^{42}$, whereas $E_{\max }$ is considered to be a measure of systolic contractility ${ }^{\sqrt{37 \mid 29140}}$. The functions $H_{1}(t)$ and $H_{2}(t)$,

$$
H_{1}(t)=\frac{\left(\bar{t} / \tau_{1}\right)^{m_{1}}}{1+\left(\bar{t} / \tau_{1}\right)^{m_{1}}} \quad \text { and } \quad H_{2}(t)=\frac{1}{1+\left(\bar{t} / \tau_{2}\right)^{m_{2}}},
$$

govern the ascending (contraction) and descending (relaxation) tracts of the $E(t)$ curve, respectively, and they were described by shape $m_{i}$ and time $\tau_{i}$ parameters, with $i=1,2$. In Eq. (2.3), $\bar{t}$ is equal to $t-t_{\text {onset }}$, with $t_{\text {onset }}$ the onset of contraction. The cavity volume $v(t)$ was calculated using the continuity equation

$$
\frac{\mathrm{d} v}{\mathrm{~d} t}=q_{\text {in }}(t)-q_{\text {out }}(t)
$$

where $q_{\text {in }}(t)$ and $q_{\text {out }}(t)$ are the flow rates entering and leaving the heart chamber.

Valve dynamics, described by the trans-valvular blood flow rate, $q(t)$, and the opening state of the valve, $\zeta(t)$, ranging from 0 (totally closed valve) to 1 (totally open valve), depends on the pressure gradient across the valve, $\Delta p(t)$. Valve dynamics over time was expressed via a system of ordinary differential equations $(\mathrm{ODE})^{25}$,

$$
\begin{aligned}
& \frac{\mathrm{d} q}{\mathrm{~d} t}=\frac{1}{L(t)}[\Delta p(t)-B(t) q(t)|q(t)|], \\
& \frac{\mathrm{d} \zeta}{\mathrm{d} t}=\mathscr{F}\left(\zeta, K_{v o}, K_{v c}, \Delta p\right)= \begin{cases}{[1-\zeta(t)] K_{v o} \Delta p(t)} & \text { if } \Delta p(t)>0, \\
\zeta(t) K_{v c} \Delta p(t) & \text { if } \Delta p(t)<0,\end{cases}
\end{aligned}
$$

where the coefficients $B(t)$ and $L(t)$ are Bernoulli resistance and blood inertance, respectively. These parameters depend on the orifice area, $A(t)$, and valve length, $l^{25}$. The orifice area ranges from $A_{\min }$ to $A_{\max }$ potentially accounting for a leaky or stenotic valve. The rate of opening or closure of the valve, $\mathrm{d} \zeta / \mathrm{d} t$, depends on the pressure gradient, such that when $\Delta p(t)$ is positive the valve opens and the coefficient $K_{v o}$ is employed; otherwise, the valve closes and $K_{v c}$ is used. High values of these coefficients indicate a rapid opening and closing 
of the valve, respectively.

115

The input to the cardiac contraction model, represented by the pulmonary venous flow rate, was prescribed as a discretized function of time. The PVFR entering the LA accounts for the flow contributions of all the pulmonary veins. It has a double-peaked shape, with two local maxima, i.e. the systolic and diastolic peaks, and two local minima, $\frac{25 / 28 / 43}{2}$ as shown in Fig. 1 (A). In this work, PVFR was parametrised using a Fourier function to allow us to make PVFR age-dependent and simulate a decreased venous return. The PVFR parametrization was accomplished by assigning six parameters: the cardiac cycle duration $T$, the total volume entering the left atrium in one cardiac cycle, $V_{n e t}$, and the four function extrema. The first five Fourier modes were used for the parametrization.

\subsubsection{D-1D coupling}

The cardiac contraction model acts as inlet boundary condition to the 1-D model vascular network. The flow rate through the aortic valve, $q_{A V}(t)$, was computed at every time step and prescribed as the inflow to the aortic root. The coupling between the cardiac model and the arterial domain was accomplished using the following Riemann invariants associated with the genuinely non-linear field at the proximal boundary of the physical domain 33 ,

$$
\Gamma_{1}=u-4 c, \quad \Gamma_{2}=p-K(\sqrt{\alpha}-1)
$$

where $u(x, t)$ is the cross-sectional averaged blood velocity, $c(x, t)$ is the pulse wave velocity, $p(x, t)$ is the cross-sectional averaged blood pressure $(\mathrm{BP}), K(x)$ is the wall stiffness coefficient, and $\alpha$ is the dimensionless luminal cross-sectional area ${ }^{3}$.

In the cardiac contraction model, the time-dependent variables presented in Sect. 2.1.2 must be computed and updated for every time step. These variables are the valve's flow rate, $q_{v}$, and state, $\zeta_{v}$, and the chambers volume, $v_{c}$. Subscript $c$ identifies either the LA or the LV, whereas subscript $v$ identifies either the MV or AV. Equations (2.5a) and 2.5b) 
were integrated in time following the IMEX RK method, treating the equations explicitly since they do not contain stiff terms. Thus, the time discretization of Eq. 2.5a reads as

$$
\begin{aligned}
q_{v}^{(k)} & =q_{v}^{n}+\Delta t \sum_{j=1}^{k-1} \tilde{a}_{k j}\left[\frac{1}{L^{(j)}}\left(\Delta p^{(j)}-B^{(j)} q^{(j)}\left|q^{(j)}\right|\right)\right], \\
q_{v}^{n+1} & =q_{v}^{n}+\Delta t \sum_{k=1}^{s} \tilde{\omega}_{k}\left[\frac{1}{L^{(k)}}\left(\Delta p^{(k)}-B^{(k)} q^{(k)}\left|q^{(k)}\right|\right)\right] .
\end{aligned}
$$

Here, $q_{v}^{n}$ is the variable at time $t^{n}$, and $q_{v}^{n+1}$ at $t^{n+1}=t^{n}+\Delta t$. Matrix $\tilde{\boldsymbol{a}}=\left(\tilde{a}_{k j}\right)$ is an $s \times s$ matrix characterizing the explicit stages of the chosen IMEX RK scheme, while the coefficient vector $\tilde{\boldsymbol{\omega}}=\left(\tilde{\omega}_{1}, \ldots, \tilde{\omega}_{s}\right)$ represents the explicit weights, with $s$ the number of the Runge-Kutta (RK) stages $(s=3 \text { in this work })^{3132}$. The same time discretization scheme was applied to the valve flow rate to obtain the explicit discretization of Eq. (2.5b)

$$
\begin{aligned}
\zeta_{v}^{(k)} & =\zeta_{v}^{n}+\Delta t \sum_{j=1}^{k-1} \tilde{a}_{k j} \mathscr{F}\left(\zeta_{v}^{(j)}, K_{v o, v}^{(j)}, K_{v c, v}^{(j)}, \Delta p_{v}^{(j)}\right), \\
\zeta_{v}^{n+1} & =\zeta_{v}^{n}+\Delta t \sum_{k=1}^{s} \tilde{\omega}_{k} \mathscr{F}\left(\zeta_{v}^{(k)}, K_{v o, v}^{(k)}, K_{v c, v}^{(k)}, \Delta p_{v}^{(k)}\right) .
\end{aligned}
$$

For the mitral valve, $\Delta p_{M V}$ is the pressure difference between the LA and the LV, whereas, for the aortic valve, $\Delta p_{A V}$ is the pressure difference between the LV and the first section of the aortic root. Heart chamber pressures were calculated using the stress-strain relationship given by Eq. 2.1). Finally, applying the IMEX RK method to Eq. 2.4), the chamber volume at each RK time step was computed using the inlet and outlet flow rates calculated at the same time step as in Eq. 2.7a), and for the final update those calculated by Eq. (2.7b), i.e.

$$
\begin{aligned}
& v_{c}^{(k)}=v_{c}^{n}+\Delta t \sum_{j=1}^{k-1} \tilde{a}_{k j}\left(q_{v, \text { in }}^{(j)}-q_{v, \text { out }}^{(j)}\right), \\
& v_{c}^{n+1}=v_{c}^{n}+\Delta t \sum_{k=1}^{s} \tilde{\omega}_{k}\left(q_{v, \text { in }}^{(k)}-q_{v, \text { out }}^{(k)}\right) .
\end{aligned}
$$

${ }_{130}$ For the LA, $q_{v, \text { in }}$ is the pulmonary venous flow rate and $q_{v, \text { out }}$ is $q_{M V}$, whereas for the LV they are $q_{M V}$ and $q_{A V}$, respectively. 
Table 1: Vascular (VV) and cardiac (CC) parameters of the numerical model. VV parameters: vessel length $(L)$, proximal and distal vessel radius $\left(r_{\text {in }}\right.$ and $r_{\text {out }}$, resp.), vessel stiffness ( $E_{Y} h$, where $E_{Y}$ is the Young's modulus and $h$ is vessel thickness) computed via Eq. (2) in ${ }^{8}$, reference pressure $\left(p_{\text {ref }}\right)$, RCR outlet pressure $\left(p_{\text {out }}\right)$, and RCR resistances and compliance $\left(R_{1}, R_{2}, C\right.$, resp.). CC parameters: total volume entering the LA in one cardiac cycle $\left(V_{n e t}\right)$, cardiac cycle duration $(T)$, minimal and maximal elastance ( $E_{\min }$ and $E_{\max }$, resp.), shape and time parameters $\left(m_{1,2}\right.$ and $\tau_{1,2}$, resp.), source resistance $\left(K_{s}\right)$, unstressed and initial volumes $\left(v_{p 0}\right.$ and $v_{0}$, resp.) and onset time of the elastance function $\left(t_{\text {onset }}\right)$; minimum and maximum valve orifice areas $\left(A_{\min }\right.$ and $A_{\max }$, resp.), valve opening and closure constants ( $K_{v o}$ and $K_{v c}$, resp.), and valve length $(l)$. Subscripts $c$ and $v$ refer to chamber and valve, respectively.

\begin{tabular}{cccccc}
\hline & $L[\mathrm{~m}]$ & $r_{\text {in }}, r_{\text {out }}[\mathrm{m}]$ & $E_{Y} h[\mathrm{~Pa} \mathrm{~m}]$ & $p_{\text {ref }}[\mathrm{Pa}]$ & $p_{\text {ext }}[\mathrm{Pa}]$ \\
$\mathrm{VV}$ & $p_{\text {out }}[\mathrm{Pa}]$ & $R_{1}\left[\mathrm{~Pa} \mathrm{~s} / \mathrm{m}^{3}\right]$ & $R_{2}\left[\mathrm{~Pa} \mathrm{~s} / \mathrm{m}^{3}\right]$ & $C\left[\mathrm{~m}^{3} / \mathrm{Pa}\right]$ & \\
\hline & $V_{\text {net }}\left[\mathrm{m}^{3}\right]$ & $E_{\text {min }, c}\left[\mathrm{~Pa} / \mathrm{m}^{3}\right]$ & $m_{1, c}[-], \tau_{1, c}[\mathrm{~s}]$ & $v_{p 0, c}\left[\mathrm{~m}^{3}\right]$ & $K_{s, c}\left[\mathrm{~s} / \mathrm{m}^{3}\right]$ \\
$\mathrm{CC}$ & $T[\mathrm{~s}]$ & $E_{\text {max }, c}\left[\mathrm{~Pa} / \mathrm{m}^{3}\right]$ & $m_{2, c}[-], \tau_{2, c}[\mathrm{~s}]$ & $v_{0, c}\left[\mathrm{~m}^{3}\right]$ & $t_{\text {onset }, c}[\mathrm{~s}]$ \\
& $A_{\text {min }, v}\left[\mathrm{~m}^{2}\right]$ & $K_{\text {vo }, v}[1 / \mathrm{Pa} \mathrm{s}]$ & $l_{v}[\mathrm{~m}]$ & & \\
& $A_{\text {max }, v}\left[\mathrm{~m}^{2}\right]$ & $K_{v c, v}[1 / \mathrm{Pa} \mathrm{s}]$ & & & \\
\hline
\end{tabular}

\subsection{Sensitivity analysis}

Given the wealth of parameters involved in the cardiovascular model, a sensitivity analysis (SA) was performed to identify those parameters that most affect blood pressure waveforms, labelled hereafter as significant. Section 2.2.1 presents the cardiovascular parameters varied in the SA, and Section 2.2.2 describes how the significant parameters were identified. Section 2.2.3 introduces the haemodynamic indices studied in the SA. 


\subsubsection{Model parameters}

The parameters defining the cardiovascular model were separated into vascular and car-

140 Appendix A.

The SA focused on the cardiac parameters, since a SA for the vascular parameters was performed by Charlton et al. $\underline{8}$.

\subsubsection{Significant parameters evaluation}

SA was performed by varying the cardiac parameters listed in Table 1 from their reference values in a univariate manner, i.e. when a parameter is varied, all others remained unchanged. Reference values of heart chamber and valve parameters are listed in Tables 2 and 3 , respectively. Reference values for $V_{\text {net }}$ and $T$ were $66.3 \mathrm{ml}$ and $0.822 \mathrm{~s}$, respectively ${ }^{8}$. Variations were performed by increasing and decreasing each reference value by the same percentage. The percentage change depends on the cardiac parameter considered:

- Cardiac parameters excluding $V_{n e t}, T, A_{m i n, A V}$, and $A_{m i n, M V}$, were varied by $\pm 50 \%$ from the reference value. Given the lack of baseline physiological variations of these modelling parameters in the literature, a $\pm 50 \%$ was chosen to investigate the effect of these parameters on pulse wave morphology. The resulting variations in LV contractility are generally within the physiological range for different clinical scenarios 27 , as shown in Sect. 3.2.2.

- The minimum orifice area of the aortic and mitral valves have a zero reference value, standing for a complete valve closure. Simulations for increased $A_{\min , A V}$ and $A_{\min , M V}$ 
were obtained by setting their values to $30 \mathrm{~mm}^{2}$, corresponding to a severe aortic regurgitant orifice area ${ }^{41}$, and to a sever mitral regurgitation ${ }^{7}$. Decreased values have no physical meaning and were not considered.

- The total volume entering the network in one cardiac cycle, $V_{n e t}$, and cardiac cycle duration, $T$, were varied as described by Charlton et al. ${ }^{8}$. Maximum and minimum values for both parameters were identified in Charlton's database. The positive and negative percentage variations from their reference value were calculated, and the greatest variation in absolute value was used in the SA. As a result, $V_{\text {net }}$ varied by $\pm 40 \%$, and $T$ by $\pm 20 \%$. The same percentage changes were used for positive and negative variations to avoid SA asymmetry.

SA simulations were compared in pairs to assess the effect on arterial pressure waveforms of changes in cardiac parameters: (i) baseline simulation and (ii) increased or decreased parameter, respectively. This comparison was made for each parameter at the aortic root, brachial artery, and digital artery. The root mean square error (RMSE) was calculated for each pair. RMSEs were found to increase towards the periphery, therefore, the cardiac parameters giving an RMSE in the digital artery above the assigned threshold of $6.5 \mathrm{mmHg}$ were labelled as significant.

\subsubsection{Haemodynamic indices}

Eleven haemodynamic indices were used in a second SA involving those cardiac parameters identified as significant in Section 2.2.2.

The following four indices of central haemodynamics were considered. Left ventricular contractility, quantified by the contractility index (CI), is the maximum rate of increase in left ventricular pressure during isovolumetric contraction, $\mathrm{d} p_{\mathrm{LV}} /\left.\mathrm{d} t\right|_{\max } 2719$. The rate of increase in flow rate and pressure at the aortic root in early systole, $\Delta q / \Delta t$ and $\Delta p / \Delta t$, respectively. $\Delta q$ was calculated as the difference between the peak flow rate, $q_{\max }$, and the flow rate at the foot of the waveform, $q_{\min }$, i.e. when the aortic valve opens. $\Delta p$ is the 
pulse pressure (PP), i.e. the difference between systolic (SBP) and diastolic (DBP) blood pressures ${ }^{[18}$. $\Delta t$ is the time interval in-between the occurrences of either $q_{\min }$ and $q_{\max }$ or DBP and SBP. Finally, the maximum rate of decrease of late-systolic flow at the aortic root, $\mathrm{d} q /\left.\mathrm{d} t\right|_{\min , A o R t}$, as described in Gerónimo et al.11.

The following four indices of cardiac function were considered: left ventricular ejection time (LVET), stroke volume (SV), left ventricular ejection fraction (EF), and cardiac output (CO). LVET is the time interval between opening and closing of the aortic valve. SV is the difference between simulated left ventricular end-diastolic volume (LVEDV) and end-systolic volume (LVESV). EF is the ratio of SV to LVEDV. Lastly, CO is equal to $\mathrm{SV} \times 60 / T$.

The following three vascular indices were considered: PP at a central (aortic root) and peripheral (digital) site, $\mathrm{PP}_{\mathrm{AoRt}}$ and $\mathrm{PP}_{\mathrm{Di}}$ respectively, and the $\mathrm{PP}$ augmentation ratio $\left(\mathrm{AR}_{\mathrm{AoRt}-\mathrm{Di}}\right)$ calculated as the percentage increase of PP between the two sites.

\section{Results}

\subsection{Cardiovascular model verification}

The vascular network model has been thoroughly tested in previous studies ${ }^{35133}$. Here, we focused on verifying the ability of the cardiac contractility model coupled to the vascular network model to produce physiological haemodynamics signals. Figure 2 compares simulated haemodynamics signals with reference in silico data, taken from Mynard and Smolich $^{28}$, and Charlton et al. ${ }^{8}$ in the heart and vasculature, respectively. These reference data were validated against in vivo measurements.

An amplitude discrepancy between simulated and reference in silico cardiac signals can be observed, especially in chamber volumes, due to different stroke volumes used in the two models $\left(83 \mathrm{ml}\right.$ in the reference model ${ }^{28}$ and $66 \mathrm{ml}$ in the new model). However, the signal morphology is well replicated by the new model, with the mitral trans-valvular flow rate presenting its characteristic double-peaked morphology 19138 . Furthermore, the state of the aortic valve variable shows a clear distinction between the LV ejection phase when the valve 

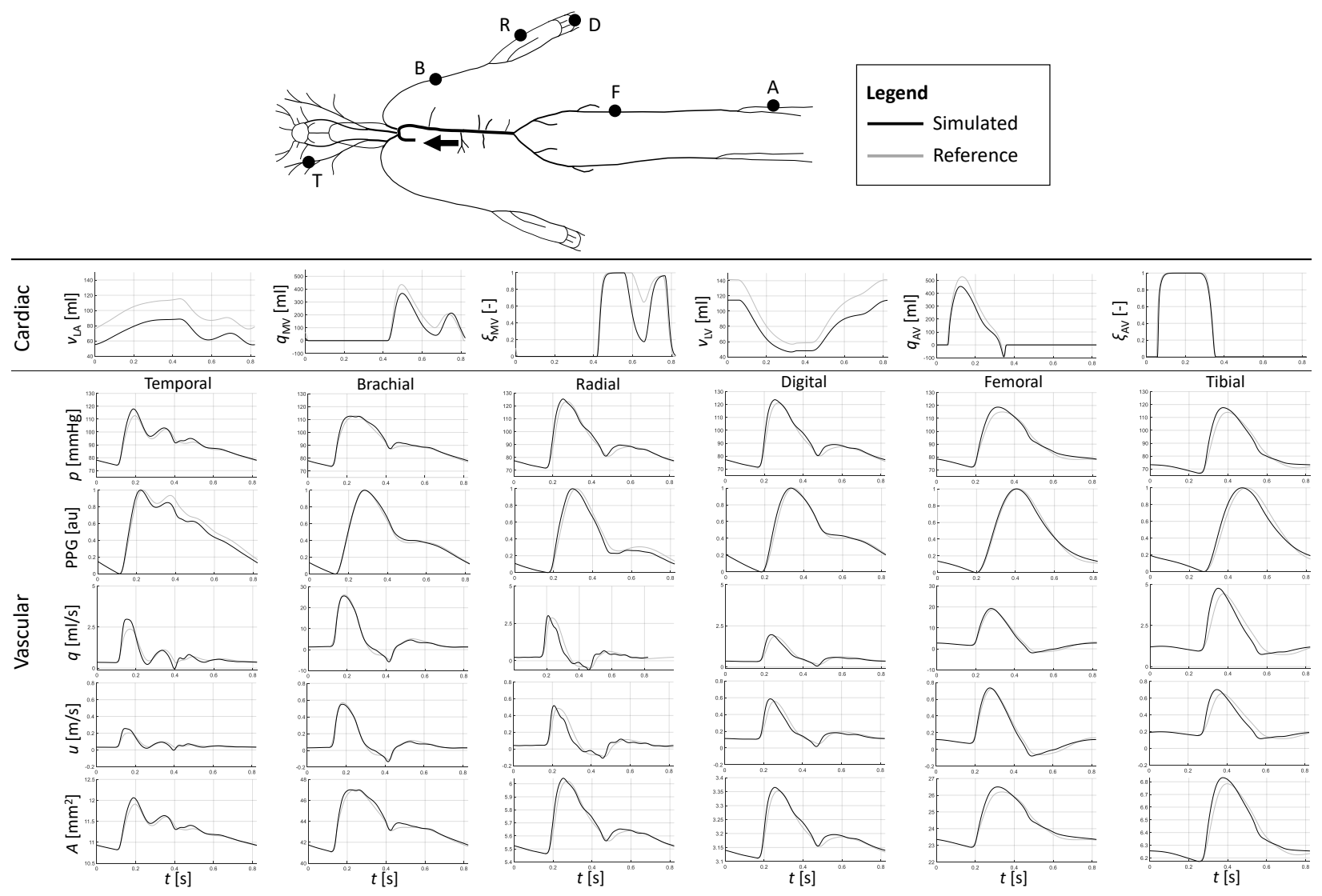

Figure 2: Haemodynamic results of the cardiovascular model. Simulated waveforms are in black solid lines, reference waveforms are in grey solid lines. Cardiac and vascular reference signals were taken from Mynard et al. $\frac{28}{}$ and Charlton at al. $\frac{8}{}$, respectively. Cardiac signals are volume in the left atrium $\left(v_{L A}\right)$, flow rate through the mitral valve $\left(q_{M V}\right)$, state of the mitral valve $\left(\zeta_{M V}\right)$, volume in the left ventricle $\left(v_{L V}\right)$, flow rate through the aortic valve $\left(q_{A V}\right)$, state of the aortic valve $\left(\zeta_{A V}\right)$. Vascular signals are internal pressure $(p(t))$, PPG signal, flow rate $(q(t))$, flow velocity $(u(t))$, and luminal cross-sectional area $(A(t))$, in the temporal, brachial, radial, digital, femoral, and tibial arteries, as indicated by the labels in the sketch on the top.

is open and the LV filling phase when it is closed. These results corroborate the ability of the cardiac contraction model to accurately simulate cardiac haemodynamics.

Figure 2 shows pulse wave signals (flow rate, flow velocity, luminal cross-sectional area, pressure, and PPG) at six arterial sites: the temporal artery in the head, three peripheral 

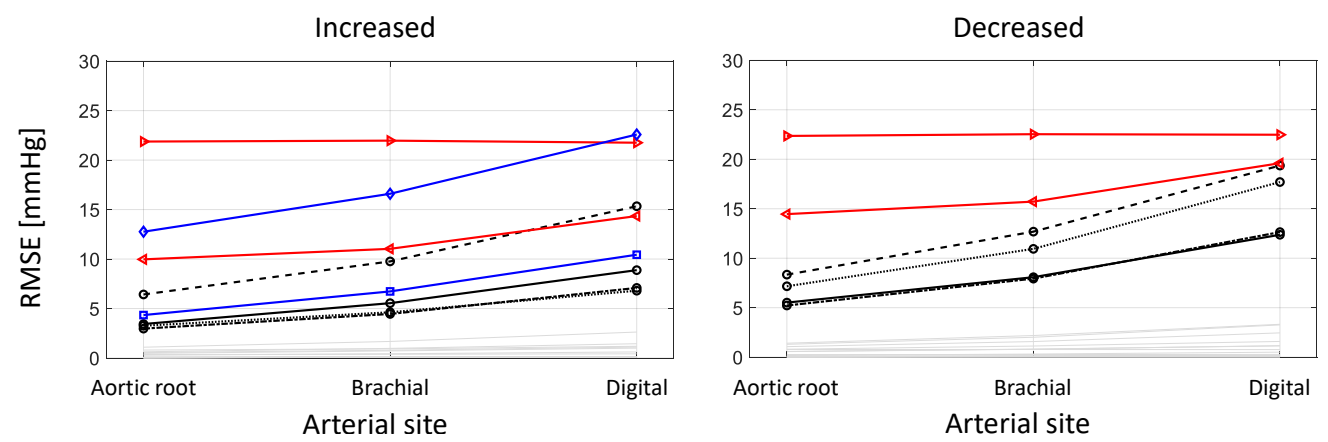

Figure 3: RMSEs computed at the aortic root, brachial and digital arteries for simulations with the increased (left) and decreased (right) cardiac parameters values. Significant parameters are divided in parameters of LV contractility (black lines), $V_{\text {net }}$ and $T$ (red lines), and heart valve-related parameters (blue lines). Grey lines represent all non-significant parameters.

sites in the arm, namely the brachial, radial, and digital arteries, and two in the leg, the femoral and the anterior tibial arteries. Reference and simulated waveforms are in excellent agreement, with percentage relative RMSEs smaller than $1 \%$ for all signals and sites, relative to reference signals.

\subsection{Sensitivity analysis}

\subsubsection{Waveform morphology}

RMSEs increased towards the periphery of the vascular network (Fig. 3), where the threshold of significant cardiac parameter was imposed (i.e. at the digital site). The resulting significant cardiac parameters were found to be the same for both scenarios in which cardiac parameters were either increased or decreased from their baseline values. These are parameters mainly attributed to LV contractility: the maximal elastance, $E_{\max , L V}$, the contraction shape parameter $m_{1, L V}$, and the contraction and relaxation time parameters, $\tau_{1, L V}$ and $\tau_{2, L V}$, respectively. The other significant parameters were the minimum orifice area of the valves, $A_{m i n, A V}$ and $A_{m i n, M V}$, the total volume entering the LA in one cardiac cycle, $V_{n e t}$, and the cardiac cycle duration, $T$. No cardiac parameters of LA contractility were found to 

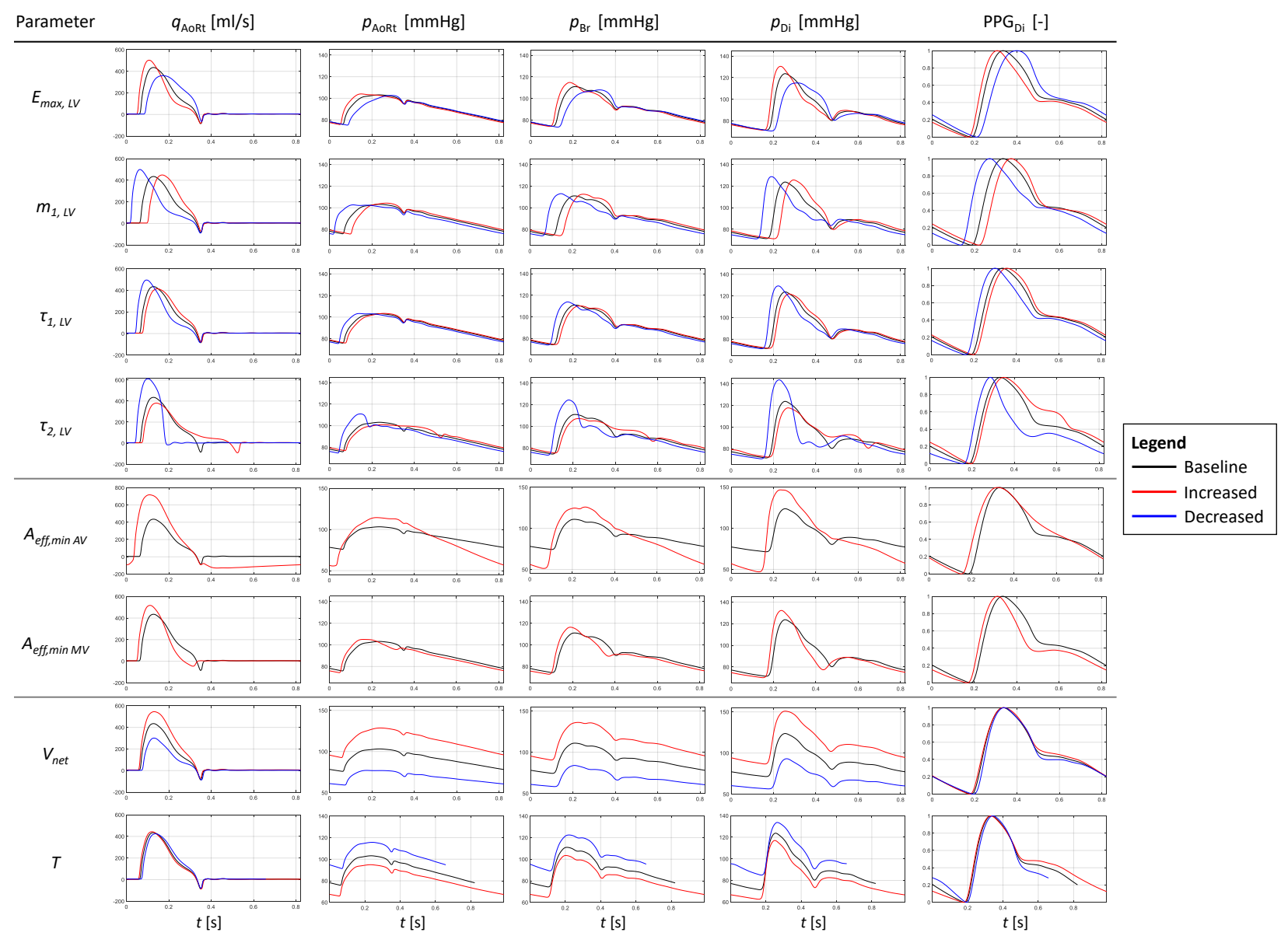

Figure 4: Pulse waveforms at baseline (black), and with increased (red) and decreased (blue) values in the significant cardiac parameters. The following waveforms are shown: flow rate at the aortic root $\left(q_{\text {AoRt }}\right)$, pressure at the aortic root $\left(p_{\text {AoRt }}\right)$, pressure in the brachial artery $\left(p_{\mathrm{Br}}\right)$, and pressure $\left(p_{\mathrm{Di}}\right)$ and $\mathrm{PPG}$ signal $\left(\mathrm{PPG}_{\mathrm{Di}}\right)$ in the digital artery. Each row represents simulations for a significant cardiac parameter: left ventricular (LV) maximal elastance $\left(E_{\text {max }, L V}\right), \mathrm{LV}$ contraction shape parameter $\left(m_{1, L V}\right), \mathrm{LV}$ contraction time parameter $\left(\tau_{1, L V}\right)$, LV relaxation time parameter $\left(\tau_{2, L V}\right)$, aortic valve minimum orifice area $\left(A_{\min , A V}\right)$, mitral valve minimum orifice area $\left(A_{m i n, M V}\right)$, total volume entering the left atrium in one cardiac cycle $\left(V_{n e t}\right)$, and cardiac cycle duration $(T)$. 
occurred mainly in systole. LV-related parameters produced minor changes in wave morphology in diastole. The timing of the dicrotic notch only changed when $\tau_{2, L V}$ was varied, since this cardiac parameter regulates the timing of the relaxation phase of LV contractility. With increasing $\tau_{2, L V}$ the relaxation phase of the LV was delayed and, hence, the aortic valve closed later, delaying the time of the dicrotic notch.

Regurgitant valve scenarios show significant variations in pulse wave morphology. With increasing $A_{m i n, A V}$ the flow rate at the aortic root became negative in diastole due to the reflux caused by the suction effect of the LV during relaxation. Consequently, arterial diastolic pressure decreased. These results suggest that impairment of the AV has direct effects on arterial pulse waves, both at central and peripheral sites. With increasing $A_{\min , M V}$ the late-systolic decrease in flow rate occurred more rapidly than in the case of a healthy valve. During LV contraction blood volume was not only ejected in the vascular network through the AV, but part of it is also re-ejected in the LA through the leaking MV. Pressures in the network with regurgitant MV changed less significantly than the AV case due to the mitigating function of the LV and the proper action of the AV.

Cardiac function, in particular LV contractility, influenced PPG morphology. Variations in PPG signals followed those in digital pressure. Interestingly, in the case of $V_{n e t}$ variations, PPG pulse waves remained unaltered due to the nearly linear relationship between volume in the network and internal pressure: when only $V_{\text {net }}$ varied, the pressure amplitude changed but not the pressure morphology.

\subsubsection{Haemodynamic indices}

Figure 5 summarises the percentage variations in haemodynamic indices with changes in significant cardiac parameters. The reference CI $\left(\mathrm{d} p_{\mathrm{LV}} /\left.\mathrm{d} t\right|_{\max }\right)$ was $13.96 \times 10^{2} \mathrm{mmHg} / \mathrm{s}$ in agreement with literature data ${ }^{279}$. The observed variations in CI are consistent with physiologic percentage variations in which CI increased by $133 \%$ during exercise and decreased by $47 \%$ with cardiomyopathy ${ }^{27}$. Most of the variations reported in Fig. 5 (A) are within this range. CI increased with increasing $E_{\max , L V}$ and decreasing $m_{1, L V}, \tau_{1, L V}$, and $\tau_{2, L V}$. 

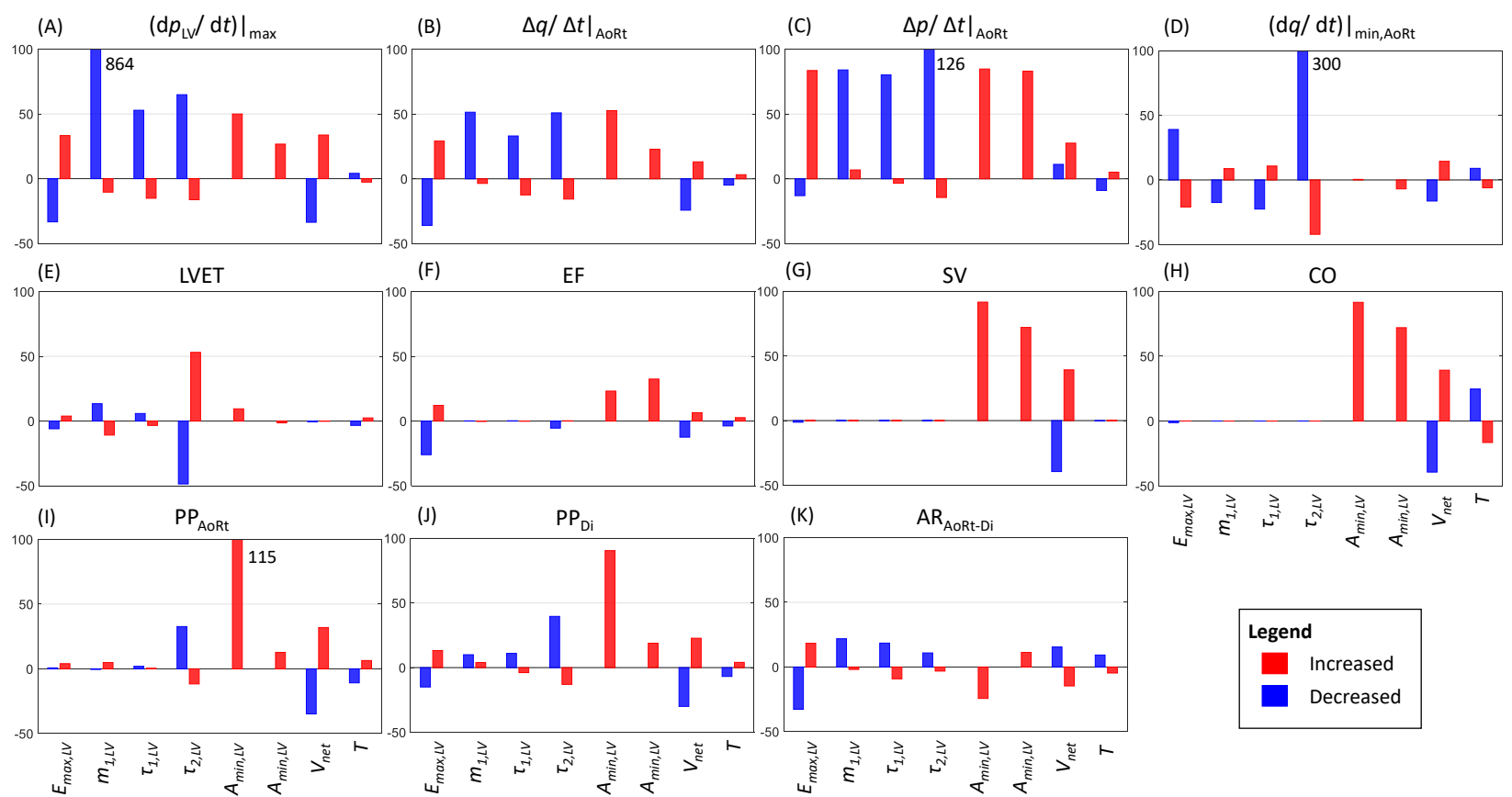

Figure 5: Percentage variations in haemodynamic indices with increased (red) and decreased (blue) values in the significant cardiac parameters. Y-axis show percentage variations in the indices from their corresponding baseline values. Baseline values: contractility index $\left(\mathrm{d} p_{\mathrm{LV}} /\left.\mathrm{d} t\right|_{\max }\right), 13.96 \times 10^{2} \mathrm{mmHg} / \mathrm{s}$, rate of increase in flow rate in early-systole at the aortic root $\left(\Delta q /\left.\Delta t\right|_{\text {AoRt }}\right), 6.45 \times 10^{3} \mathrm{ml} / \mathrm{s}^{2}$, rate of increase in pressure in early-systole at the aortic root $\left(\Delta p /\left.\Delta t\right|_{\text {AoRt }}\right), 1.47 \times 10^{2} \mathrm{mmHg} / \mathrm{s}$, maximum rate of decrease of late-systolic flow rate at the aortic root $\left(\mathrm{d} q /\left.\mathrm{d} t\right|_{\min , \text { AoRt }}\right), 2.42 \times 10^{3} \mathrm{ml} / \mathrm{s}^{2}$, left ventricular ejection time (LVET), $324 \mathrm{~ms}$, ejection fraction (EF), 58 \%, stroke volume (SV), $66.4 \mathrm{ml}$, cardiac output $(\mathrm{CO}), 4.8 \mathrm{l} / \mathrm{min}$, pulse pressure at the aortic root $\left(\mathrm{PP}_{\text {AoRt }}\right), 27.3 \mathrm{mmHg}$, pulse pressure in the digital artery $\left(\mathrm{PP}_{\mathrm{Di}}\right), 52.3 \mathrm{mmHg}$, and pulse pressure augmentation ratio between the aortic root and the digital artery $\left(\mathrm{AR}_{\mathrm{AoRt}-\mathrm{Di}}\right), 91.8 \%$.

The most significant variation in CI occurred with the decreasing shape parameter $m_{1, L V}$, which defines the slope of the increasing part of the chamber elastance curve. A smaller $m_{1, L V}$ made the slope steeper and hence the rate of increase of $p_{\mathrm{LV}}$. Impaired valves and $V_{\text {net }}$ caused an increase in CI, whereas $T$ led to negligible effects on CI. $\Delta q / \Delta t$ and $\Delta p / \Delta t$ at the aortic root varied similarly to variations in CI, although at different rates (Fig. 5 (B) and 
(C)). The relation between increased LV contractility and aortic root pulse wave morphology

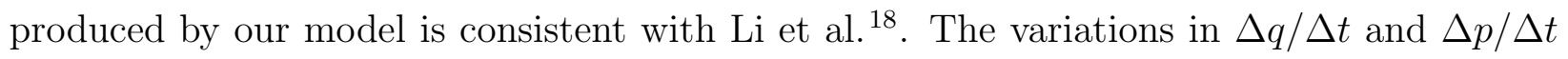
followed the same trend as those in $\mathrm{PP}_{\mathrm{Di}}($ Fig. $5(\mathrm{~J})$ ), in agreement with the morphology of the central pressure wave being a major determinant of peripheral SBP and correlating with the rate of increase in early-systolic aortic flow 18111 . Moreover, the maximum rate of decrease in late-systolic flow at the aortic root, $\mathrm{d} q /\left.\mathrm{d} t\right|_{\min , \text { AoRt }}$ in Fig. 5 (D), increased with increasing $V_{\text {net }}$, and decreasing $T$ and LVET, in agreement with Flores et al. ${ }^{11}$ As for LV properties, both EF and LVET were affected by changes in LV contractility, as shown in Fig. 5 (E) and (F). EF also increased with regurgitant valves and increasing $V_{\text {net }}$ and $T$. The variation in LVET became significant when $\tau_{2, L V}$ was varied: it decreased by ${ }_{280}-48 \%$ with decreasing $\tau_{2, L V}$ and increased by $+52 \%$ with increasing $\tau_{2, L V}$. This result is consistent with the physical meaning of the contraction and relaxation time parameters. These influence the time of occurrence of the respective sections of the elastance curve: a decrease in $\tau_{1, L V}$ anticipates contraction and an increase in $\tau_{2, L V}$ delays relaxation, and viceversa. In the case of AV regurgitation, LVET is the time interval when the area of the $\mathrm{AV}$ is greater than its minimum value. SV was not affected by the four cardiac parameters related to LV contractility (Fig. $5(\mathrm{G})$ ), since $V_{\text {net }}$ did not change. A significant increase in SV can be observed when simulated valve impairment scenarios, of $+92 \%$ and $+72 \%$ for the AV and MV, respectively. When the AV does not close completely, part of the volume ejected during the contraction returns into the ventricle during relaxation, as pointed out in Sect. 3.2. This causes both the LV end-diastolic and end-systolic volumes to increase, with a greater increase in the former than the latter, thus resulting in an overall increase in SV. Similarly, the LV ejects blood volume through both valves when the MV is impaired. Thus, the LV end-systolic volume decreases and the end-diastolic volume increases. Variations in $V_{\text {net }}$ caused SV to change symmetrically from the baseline value, demonstrating that, barring changes in other cardiac parameters, $V_{\text {net }}$ is a surrogate of stroke volume. As expected, $\mathrm{CO}$ in Fig. $5(\mathrm{H})$ changed consistently with SV and $T$, most significantly influenced by the former. 
The pulse pressure PP showed less variation in central sites compared to the periphery (Fig. $5(\mathrm{I})$ and $(\mathrm{J})$ ). PP increased with increasing LV contractility, i.e. higher peak and rate of 300 servable from variations in $\tau_{2, L V}$. Regurgitant valves and increased $V_{\text {net }}$ and $T$ also raised PP. Greater contractility augmented $\mathrm{AR}_{\mathrm{AoRt}-\mathrm{Di}}$ (Fig. $5(\mathrm{~K})$ ), hence enhancing the physiological effect of PP amplification towards peripheral sites $\frac{8 \mid 18}{}$. AR $\mathrm{AoRt}_{\mathrm{Ai}}$ decreased with impaired $\mathrm{AV}$, caused by the greater increase in $\mathrm{PP}_{\text {AoRt }}$ compared to $\mathrm{PP}_{\mathrm{Di}}$ from their baseline values.

\section{Discussion}

This work has studied the relationship between cardiac properties and vascular haemodynamics using a state-of-the-art 1-D model. It has been demonstrated that the presented cardiovascular model correctly reproduces human haemodynamics, consistent with in vivo measurements. The sensitivity analysis has shown that a variation in each cardiac property is associated with an identifiable variation in waveform morphology, suggesting the possibility of applying techniques for inverse problem solving, going from a specific pressure waveform to the heart characteristics that generated it. Moreover, the contractility of the LA has negligible effects on vascular pulse waves, suggesting that LA properties can not be derived from a pulse wave analysis. In contrast, LV contractility, stroke volume, cardiac cycle duration, and impaired valves function have a considerable influence on arterial pulse waves, being determinants of central waveforms morphology, pulse pressure and its amplification. The greatest variations in PP were found at peripheral sites rather than central sites, which may be more valuable sites for extracting information about cardiac function by pulse wave analysis. Furthermore, our results have corroborated the findings of previous studies showing strong correlations between LV contractility, aortic blood flow and PP 11118 , and the crucial role of cardiac function in both central and peripheral pressure amplitudes $\frac{12[44}{\text {. }}$

Our results have shown that LV contractility, measured by CI, is related to peak elastance, $E_{\max , L V}$, rate of LV contraction, and LVET. An increase in $E_{\max , L V}$ allows the LV pressure 
to reach higher values, as $E_{\max , L V}$ and LV pressure are directly related through the strainstress relationship of the cardiac chamber. CI increases with a faster contraction simulated by decreased $m_{1, L V}$, and time parameters. As $\tau_{1, L V}$ decreases, LV pressure increases more rapidly, and so does pressure and flow rate at the aortic root. Moreover, the decrease in $\tau_{2, L V}$ results in a shortening of LVET, leading to the same SV being ejected in a shorter time inteval, making the ejection more impulsive. The increase in contractility correlates with the increase in the $\mathrm{PP}$ augmentation ratio, i.e. PP increases more in the periphery than centrally (Fig. 5(A) and (K)). Furthermore, increased CI is associated with greater SBP peaks in the waveforms (see Fig. 4). This phenomenon is generally more pronounced towards the periphery, and is evident in the case of decreased $\tau_{2, L V} \cdot V_{\text {net }}$, a surrogate for $\mathrm{SV}$, and cardiac cycle duration, $T$, both affected blood pressure in the vascular network. With increasing $V_{\text {net }}$ and $T$, PP increased at both the central and peripheral sites, but the PP augmentation ratio decreased from the baseline simulation. Results have shown that valve impairment affects pulse waves, particularly in the case of a regurgitant AV due to its direct coupling with the vascular network. In particular, a regurgitant AV affects more significantly central sites than peripheral sites, due to a reasonable dampening effect of the vascular network. Further research is needed to investigate the correlation between valve disease and peripheral pressure in a large cohort of in vivo subjects with valve dysfunction via non-invasive measurements of peripheral BP.

Some aspects of the presented cardiovascular model should be underlined. First, some cardiac parameters that characterise the LV contractility and describe the elastance function cannot be measured directly. Therefore, it is cumbersome to define a physiological range of values for these parameters. Second, cardiac parameters affect vascular haemodynamics independently of each other, enabling the study of the effect on pulse wave morphology of independent changes in cardiac properties. This is not the case in vivo, where it would be challenging to change LV contractility, for example through pharmacological intervention, without affecting the properties of the vasculature, and vice-versa.

We have presented a theoretical, computationally-based approach to arouse interest in as- 


\section{Conflict of Interest}

No conflict of interest.

sessing cardiac function from vascular pressure waves or peripheral PPGs. For example, this work suggests that it may be possible to infer LV dysfunction, such as an impaired relaxation phase, by analysing the timing of the dicrotic notch. Results show that the time of diastole of the pulse waveform only varies with the relaxation time parameter of the LV elastance function, but is not affected by other cardiac parameters. This study also offers valuable insight into in the field of hypertension. Lately, the importance of LV contractility in hypertension has been studied, showing that LV function is a major determinant in blood pressure elevation $12 / 44|17| 11 / 18$. Our results support this thesis, as PP increased with increasing LV contractility. Moreover, hypertension has been found to be related to left ventricular hypertrophy, a response to chronic pressure overload ${ }^{\sqrt[36]{6}}$, and heart valves disease $\mathrm{e}^{3016 \sqrt[24]{24}}$. Assessing cardiac properties from pulse wave analysis in the cardiovascular network, although a challenging task, may allow cardiac function to be assessed without the need for invasive catheterization. More accessible measurements in peripheral arteries could be employed, such as in the radial artery, or one of its surrogates, the digital artery 23 .

Finally, our results have highlighted the importance of studying PPG signals, as already presented in previous works in which machine learning models were employed to determine

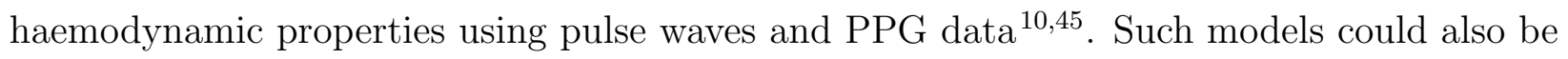
applied to infer cardiac properties from peripheral pressure measurements and PPG signals. Given the popularity of wearable devices, such as FitBits, smartwatches and oxymetries that are able to measure PPG signals, understanding their correlation with cardiac function could provide consumers with personal tools for monitoring cardiac function. 


\section{${ }_{375}$ Aknowledgements}

AV was funded by FIR 2020 of the University of Ferrara. VC was funded by MIUR FFABR 2017. JA acknowledges support from the EPSRC [EP/K031546/1], the Wellcome/EPSRC Centre for Medical Engineering at King's College London [WT 203148/Z/16/Z], the Department of Health through the National Institute for Health Research (NIHR) Car380 diovascular MedTech Co-operative at Guy's and St Thomas' NHS Foundation Trust (GSTT), and the comprehensive Biomedical Research Centre and Clinical Research Facilities awards to Guy's and St Thomas' NHS Foundation Trust in partnership with King's College London and King's College Hospital NHS Foundation Trust. 
Table 2: Reference parameters of the cardiac contraction model for the heart chambers: left atrium (LA) and left ventricle (LV).

\begin{tabular}{lcccccccccc}
\hline & $E_{\text {min }}$ & $E_{\text {max }}$ & $K_{S}$ & $v_{p 0}$ & $v_{0}$ & $m_{1}$ & $\tau_{1}$ & $m_{2}$ & $\tau_{2}$ & $t_{\text {onset }}$ \\
Chamber & {$\left[\frac{\mathrm{Pa}}{\mathrm{cm}^{3}}\right]$} & {$\left[\frac{\mathrm{Pa}}{\mathrm{cm}^{3}}\right]$} & {$\left[\frac{\mathrm{s}}{\mathrm{cm}^{3}}\right]$} & {$\left[\mathrm{cm}^{3}\right]$} & {$\left[\mathrm{cm}^{3}\right]$} & {$[-]$} & {$[\mathrm{s}]$} & {$[-]$} & {$[\mathrm{s}]$} & {$[\mathrm{s}]$} \\
\hline LA & 12.0 & 17.3 & 250 & 3.00 & 7.10 & 1.99 & 0.042 & 11.2 & 0.138 & 0.00 \\
LV & 9.33 & 373 & 500 & 1.00 & 136 & 1.32 & 0.215 & 21.9 & 0.362 & 0.65 \\
\hline
\end{tabular}

Table 3: Reference parameters of the cardiac contraction model for the heart valves: mitral valve $(\mathrm{MV})$ and aortic valve $(\mathrm{AV})$. The reference $A_{\min }$ is equal to zero for both valves.

\begin{tabular}{ccccc}
\hline & $\begin{array}{c}A_{\text {max }} \\
\text { Valve }\end{array}$ & $l$ & $K_{\text {vo }}$ & $K_{\text {vo }}$ \\
{$\left[\mathrm{cm}^{2}\right]$} & {$[\mathrm{cm}]$} & {$\left[\mathrm{Pa}^{-1} \mathrm{~s}^{-1}\right]$} & {$\left[\mathrm{Pa}^{-1} \mathrm{~s}^{-1}\right]$} \\
\hline MV & 5.1 & 2.0 & 0.2 & 0.4 \\
AV & 4.9 & 1.0 & 0.2 & 0.2 \\
\hline
\end{tabular}

\section{A Sensitivity Analysis: reference parameters}

Tables 2 and 3 show the reference cardiac parameters ${ }^{28}$ that were increased by $+50 \%$ and decreased by - $50 \%$ in the sensitivity analysis.

\section{B Sensitivity Analysis: RMSE}

Table 4 shows the RMSE evaluated in the three sites of analysis in the network, i.e. the aortic root (AoRt), the brachial artery (Br), and the digital artery (Di). Results are reported 390 for each cardiac parameter and for both variations, i.e $\pm 50 \%$. 
Table 4: Root mean square errors (RMSE) in mmHg resulted from the sensitivity analysis performed in an univariate manner. The Increased and Decreased columns stand for the simulations with the increased or decreased cardiac parameter, respectively. The amount of variation for each parameter is addressed in Sect 2.2.2. Cardiac parameters are classified per cardiac site, namely left ventricle (LV), left atrium (LA), aortic valve (AV), and mitral valve (MV). The total volume entering the LA in a cardiac cycle, $V_{n e t}$, and the cardiac cycle duration $T$ are not attributed to any cardiac site because they describe overall cardiac function. Significant parameters are reported in bold.

\begin{tabular}{|c|c|c|c|c|c|c|c|}
\hline \multirow{2}{*}{ Cardiac site } & \multirow{2}{*}{ Param. } & \multicolumn{3}{|c|}{ Increased } & \multicolumn{3}{|c|}{ Decreased } \\
\hline & & AoRt & $\mathrm{Br}$ & $D i$ & AoRt & $B r$ & $D i$ \\
\hline \multirow{2}{*}{-} & $V_{\text {net }}$ & 21.9 & 22.0 & 21.8 & 22.4 & 22.5 & 22.5 \\
\hline & $T$ & 10.0 & 11.0 & 14.3 & 14.5 & 15.7 & 19.6 \\
\hline \multirow{8}{*}{$\mathrm{LV}$} & $E_{\max }$ & 3.43 & 5.54 & 8.88 & 5.51 & 8.08 & 12.4 \\
\hline & $E_{m i n}$ & 0.80 & 0.92 & 1.19 & 0.58 & 0.82 & 1.14 \\
\hline & $m_{1}$ & 6.42 & 9.76 & 15.3 & 8.34 & 12.7 & 19.4 \\
\hline & $\tau_{1}$ & 2.97 & 4.45 & 7.08 & 5.22 & 7.94 & 12.6 \\
\hline & $m_{2}$ & 0.63 & 0.97 & 1.44 & 1.41 & 2.19 & 3.32 \\
\hline & $\tau_{2}$ & 3.26 & 4.62 & 6.80 & 7.17 & 10.9 & 17.7 \\
\hline & $v_{p_{0}}$ & 0.07 & 0.10 & 0.14 & 0.06 & 0.09 & 0.12 \\
\hline & $K_{s}$ & 1.09 & 1.67 & 2.62 & 1.30 & 2.01 & 3.26 \\
\hline \multirow{8}{*}{ LA } & $E_{\max }$ & 0.35 & 0.38 & 0.42 & 0.78 & 0.78 & 0.77 \\
\hline & $E_{m i n}$ & 0.03 & 0.09 & 0.14 & 0.04 & 0.04 & 0.04 \\
\hline & $m_{1}$ & 0.02 & 0.08 & 0.12 & 0.04 & 0.09 & 0.13 \\
\hline & $\tau_{1}$ & 0.02 & 0.07 & 0.10 & 0.03 & 0.03 & 0.03 \\
\hline & $m_{2}$ & 0.03 & 0.09 & 0.14 & 0.01 & 0.06 & 0.10 \\
\hline & $\tau_{2}$ & 0.05 & 0.09 & 0.12 & 0.26 & 0.27 & 0.28 \\
\hline & $v_{p_{0}}$ & 0.07 & 0.09 & 0.14 & 0.03 & 0.03 & 0.03 \\
\hline & $K_{s}$ & 0.02 & 0.07 & 0.10 & 0.04 & 0.09 & 0.13 \\
\hline \multirow{5}{*}{$\mathrm{AV}$} & $A_{\max }$ & 0.55 & 0.78 & 1.10 & 1.06 & 1.58 & 2.46 \\
\hline & $A_{m i n}$ & 12.8 & 16.6 & 22.6 & - & - & - \\
\hline & $l$ & 0.47 & 0.72 & 1.0 & 0.56 & 0.80 & 1.16 \\
\hline & $K_{v o}$ & 0.09 & 0.09 & 0.15 & 0.17 & 0.32 & 0.51 \\
\hline & $K_{v c}$ & 0.29 & 0.43 & 0.61 & 0.80 & 1.13 & 1.59 \\
\hline \multirow{5}{*}{ MV } & $A_{\max }$ & 0.09 & 0.12 & 0.15 & 0.13 & 0.13 & 0.13 \\
\hline & $A_{m i n}$ & 4.34 & 6.73 & 10.4 & - & - & - \\
\hline & $l$ & 0.04 & 0.09 & 0.13 & 0.04 & 0.09 & 0.14 \\
\hline & $K_{v o}$ & 0.03 & 0.07 & 0.11 & 0.04 & 0.09 & 0.12 \\
\hline & $K_{v c}$ & 0.02 & 0.07 & 0.11 & 0.02 & 0.07 & 0.11 \\
\hline
\end{tabular}




\section{References}

1. Ambrosi, D., A. Quarteroni, and G. Rozza. Modeling of Physiological Flows. Springer, 2012.

2. Bertaglia, G., V. Caleffi, L. Pareschi, and A. Valiani. Uncertainty quantification of viscoelastic parameters in arterial hemodynamics with the a-FSI blood flow model. Journal of Computational Physics, 430:110102, 2021.

3. Bertaglia, G., V. Caleffi, and A. Valiani. Modeling blood flow in viscoelastic vessels: the 1D augmented fluid-structure interaction system. Computer Methods in Applied Mechanics and Engineering, 360:112772, 2020.

4. Bertaglia, G., M. Ioriatti, A. Valiani, M. Dumbser, and V. Caleffi. Numerical methods for hydraulic transients in visco-elastic pipes. Journal of Fluids and Structures, 81:230-254, 2018 .

5. Bertaglia, G., A. Navas-Montilla, A. Valiani, M. I. Monge García, J. Murillo, and V. Caleffi. Computational hemodynamics in arteries with the one-dimensional augmented fluid-structure interaction system: viscoelastic parameters estimation and comparison with in-vivo data. Journal of Biomechanics, 100:109595, 2020.

6. Bonow, R. O. Chronic mitral regurgitation and aortic regurgitation: Have indications for surgery changed? Journal of the American College of Cardiology, 61(7):693-701, 2013.

7. Carabello, B. A. Progress in mitral and aortic regurgitation. Progress in Cardiovascular Diseases, 43(6):457-475, 2001.

8. Charlton, P. H., J. Mariscal Harana, S. Vennin, Y. Li, P. Chowienczyk, and J. Alastruey. Modeling arterial pulse waves in healthy aging: a database for in silico evaluation of hemodynamics and pulse wave indexes. American journal of physiology. Heart and circulatory physiology, 317(5):H1062-H1085, 2019. 
9. Chengode, S. Left ventricular global systolic function assessment by echocardiography. Annals of Cardiac Anaesthesia, 19(5):S26-S34, 2016.

10. Elgendi, M. PPG Signal Analysis: An Introduction Using MATLAB. CRC Press, Boca Raton, first edit edition, 2020.

11. Flores Gerónimo, J., E. Corvera Poiré, P. Chowienczyk, and J. Alastruey. Estimating Central Pulse Pressure From Blood Flow by Identifying the Main Physical Determinants of Pulse Pressure Amplification. Frontiers in Physiology, 12(February):1-15, 2021.

12. Gaddum, N., J. Alastruey, P. Chowienczyk, M. C. Rutten, P. Segers, and T. Schaeffter. Relative contributions from the ventricle and arterial tree to arterial pressure and its amplification: An experimental study. American Journal of Physiology - Heart and Circulatory Physiology, 313(3):H558-H567, 2017.

13. Gutierrez-Lemini, D. Engineering viscoelasticity. Springer, 2014.

14. Huttunen, J. M., L. Kärkkäinen, M. Honkala, and H. Lindholm. Deep learning for prediction of cardiac indices from photoplethysmographic waveform: A virtual database approach. International Journal for Numerical Methods in Biomedical Engineering, 36(3):1-17, 2020.

15. Jin, W. and J. Alastruey. Arterial pulse wave propagation across stenoses and aneurysms: Assessment of one-dimensional simulations against three-dimensional simulations and in vitro measurements. Journal of the Royal Society Interface, 18(177), 2021.

16. Lakes, R. Viscoelastic Materials. Cambridge University Press, 2009.

17. Li, Y., H. Gu, H. Fok, J. Alastruey, and P. Chowienczyk. Forward and Backward Pressure Waveform Morphology in Hypertension. Hypertension, 69(2):375-381, 2017.

18. Li, Y., A. Guilcher, P. H. Charlton, S. Vennin, J. Alastruey, and P. Chowienczyk. Relationship between fiducial points on the peripheral and central blood pressure waveforms: 
rate of rise of the central waveform is a determinant of peripheral systolic blood pressure. American journal of physiology. Heart and circulatory physiology, 320(4):H1601-H1608, 2021.

19. Luisada, A. A., K. Watanabe, P. K. Bhat, D. B. Rao, and V. Knighten. Correlates of the Echocardiographic Waves of the Mitral Valve in Normal Subjects of Various Ages. Journal of the American Geriatrics Society, 23(5):216-223, 1975.

20. Maksuti, E., N. Westerhof, B. E. Westerhof, M. Broomé, and N. Stergiopulos. Contribution of the arterial system and the heart to blood pressure during normal aging - A simulation study. PLoS ONE, 11(6):1-12, 2016.

21. Matthys, K. S., J. Alastruey, J. Peiró, A. W. Khir, P. Segers, P. R. Verdonck, K. H. Parker, and S. J. Sherwin. Pulse wave propagation in a model human arterial network: Assessment of 1-D numerical simulations against in vitro measurements. Journal of Biomechanics, 40(15):3476-3486, 2007.

22. McEniery, C. M., J. R. Cockcroft, M. J. Roman, S. S. Franklin, and I. B. Wilkinson. Central blood pressure: Current evidence and clinical importance. European Heart Journal, 35(26):1719-1725, 2014.

23. Millasseau, S. C., F. G. Guigui, R. P. Kelly, K. Prasad, J. R. Cockcroft, J. M. Ritter, and P. J. Chowienczyk. Noninvasive assessment of the digital volume pulse: Comparison with the peripheral pressure pulse. Hypertension, 36(6):952-956, 2000.

24. Mrsic, Z., S. P. Hopkins, J. L. Antevil, and P. S. Mullenix. Valvular Heart Disease. Primary Care - Clinics in Office Practice, 45(1):81-94, 2018.

25. Mynard, J. P. Computer modeling and wave intensity analysis of perinatal cardiovacular fucntion and dysfunction. Phd thesis, University of Melbourne, 2011.

26. Mynard, J. P., A. Kondiboyina, R. Kowalski, M. M. H. Cheung, and J. J. Smolich. 
Measurement, Analysis and Interpretation of Pressure / Flow Waves in Blood Vessels. Frontiers in Physiology, 2020.

27. Mynard, J. P. and P. Nithiarasu. A 1D arterial blood flow model incorporating ventricular pressure, aortic valve and regional coronary flow using the locally conservative Galerkin (LCG) method. Communication in Numerical Methods in Engineering, 24(5):367-417, 2008.

28. Mynard, J. P. and J. J. Smolich. One-Dimensional Haemodynamic Modeling and Wave Dynamics in the Entire Adult Circulation. Annals of Biomedical Engineering, 43(6):1443-1460, 2015.

29. Mynard, J. P., J. J. Smolich, M. R. Davidson, and D. Penny. A simple, versatile valve model for use in lumped parameter and one-dimensional cardiovascular models. International Journal for Numerical Methods in Biomedical Engineering, 28(6-7)::626-41, 2011.

30. Pai, R. G. and P. Varadarajan. Prognostic implications of mitral regurgitation in patients with severe aortic regurgitation. Circulation, 122(11 SUPPL. 1):43-47, 2010.

31. Pareschi, L. and G. Russo. Implicit-explicit Runge-Kutta schemes for stiff systems of differential equations. Recent trends in numerical analysis, 3(5):269-288, 2001.

32. Pareschi, L. and G. Russo. Implicit-explicit Runge-Kutta schemes and applications to hyperbolic systems with relaxation. Journal of Scientific Computing, 25(1):129-155, 2005 .

33. Piccioli, F., G. Bertaglia, A. Valiani, and V. Caleffi. Modeling blood flow in networks of viscoelastic vessels with the 1D augmented fluid-structure interaction system. Preprint. arXiv:2101.12614, 2021 (Submitted).

34. Reddy, Y. N. and R. A. Nishimura. Hemodynamics for the structural interventionalist. Elsevier, Philadelphia, 2021. 
35. Reymond, P., F. Merenda, F. Perren, D. Rüfenacht, and N. Stergiopulos. Validation of a one-dimensional model of the systemic arterial tree. American Journal of Physiology - Heart and Circulatory Physiology, 297(1):208-222, 2009.

36. Segers, P., N. Stergiopulos, J. J. Schreuder, B. E. Westerhof, and N. Westerhof. Left ventricular wall stress normalization in chronic pressure-overloaded heart: A mathematical model study. American Journal of Physiology - Heart and Circulatory Physiology,

43. Sun, Y., B. J. Sjoberg, P. Ask, D. Loyd, and B. Wranne. Mathematical model that characterizes transmitral and pulmonary venous flow velocity patterns. American Journal of Physiology - Heart and Circulatory Physiology, 268(1 37-1), 1995. 
44. Vennin, S., Y. Li, M. Willemet, H. Fok, H. Gu, P. Charlton, J. Alastruey, and P. Chowienczyk. Identifying hemodynamic determinants of pulse pressure: A combined numerical and physiological approach. Hypertension, 70(6):1176-1182, 2017.

45. Wang, T., W. Jin, F. Liang, and J. Alastruey. Machine learning-based pulse wave analysis for early detection of abdominal aortic aneurysms using in silico pulse waves. Symmetry, 13(5), 2021.

46. Willemet, M., S. Vennin, and J. Alastruey. Computational assessment of hemodynamicsbased diagnostic tools using a database of virtual subjects: Application to three case studies. Journal of Biomechanics, 49(16):3908-3914, 2016.

47. Xiao, N., J. Alastruey, and C. A. Figueroa. A systematic comparison between 1-D and 3-D hemodynamics in compliant arterial models. International Journal for Numerical Methods in Biomedical Engineering, 30(2):204-231, 2014. 